\title{
Untersuchungen am Mycobacterium tuberculosis
}

\author{
III. Mitt.: Licht- und elektronenmikroskopischer Nachweis des extrazellulären Auftretens \\ von Granula und Metaphosphatkörnchen
}

\author{
Von Hérmann KöLbel \\ Aus dem Tuberkulose-Forschungsinstitut Borstel, Institut für experimentelle Biologie \\ und Medizin (Direktor: Prof. Dr. Dr. E. Fr e e r k s e n) \\ (Z. Naturforschg. 10 b, 433-436 [1955]; eingegangen am 11. Februar 1955)
}

\begin{abstract}
An Hand von Phasenkontrast-Serienaufnahmen wird gezeigt, daß die den Granula gleichzusetzenden Mitochondrien aus der Bakterienzelle austreten können. Ihr extrazelluläres Auftreten wird durch e-optische Aufnahmen belegt. Die häufig in großer Zahl produzierten und in Freiheit gesetzten Mitochondrien werden in unmittelbarer Nähe von lysierenden Bakterien, aber auch frei im Medium angetroffen. Bei der Diskussion der biologischen Bedeutung dieses Vorganges muß berücksichtigt werden, daß die Mitochondrien Hauptsitz der Zellfermente sind und diese auch außerhalb der zellulären Organisation zu abbauenden und synthetisierenden Leistungen befähigt sind. Wie verschiedene Beobachtungen nahelegen, kann das Nährsubstrat durch die Fermentaktivität freier Mitochondrien eine Änderung erfahren, welche die übrigen Keime der Population wachstums-stimulierend beeinflussen. Andere Befunde deuten darauf hin, daß die Granula auch das Tuberkulin enthalten; doch ist die Zuordnung der vielfältig gefundenen Stoffkomplexe zu den Granula noch nicht mit Sicherheit möglich.
\end{abstract}

$\mathrm{Na}$ ach der zur Zeit herrschenden Auffassung sind die „Granula“ des Mycobacterium tuberculosis Mitochondrien ( $\mathrm{M} \mathrm{udd}$ und Mitarbb. ${ }^{1}$ ). Sie entsprechen in ihrer Funktion den von höheren Organismen bekannten Zellorganellen. Ihre elektronenoptische Abbildbarkeit rührt in erster Linie von der Einlagerung von Metaphosphat her (R u ska und Mitarbb. ${ }^{2}$ ). Die lichtmikroskopischen Granulakomplexe können nicht mit den elektronenoptisch sichtbaren Metaphosphatkörnchen identifiziert werden $\left(\mathrm{K}_{\mathrm{O}} \mathrm{lb} \mathrm{e} \mathrm{l}^{3}\right.$ ); doch scheint eine Bildung und Anreicherung von Metaphosphat nur innerhalb der lichtmikroskopisch als Mitochondrien erkannten Komplexe möglich. Da die Mitochondrien als Träger struktur-gebundener Fermente gelten, kommt einem isolierten, extrazellulären Auftreten eine besondere Bedeutung zu.

Wir verwendeten für unsere Untersuchungen einen Laboratoriumsstamm von Mycobacterium avium (M. tuberculosis, Typ. gallinaceus). Um möglichst homogenes Material zu erhalten, wurden die Bakterien von etwa 14 Tage alten Petragnani-Kulturen überimpft. Die Bilder haben wir von Bakterien gewonnen, welche im flüssigen Nährsubstrat nach Dubos ohne besondere Zusätze gezüchtet wurden. Die Kulturen waren zumeist nur wenige Tage alt und wiesen außer wenigen lysierenden völlig intakte und sich vermehrende Bakterien auf.

1 St. Mudd, L. C. Winterscheid, E. D. De L a m a ter u. H. J. Henders on, J. Bacteriol. 62, 459 [1951].
Auf der in den Abb. $1^{*}-6$ wiedergegebenen Bildserie ist zu erkennen, daß nach anfänglichem Dickenund Längenwachstum sich seitlich am Bakterium eine Ausstülpung bildet, die bald darauf platzt. Mit dem plasmatischen Material werden auch einige große Granula entlassen; die kleineren sind lichtmikroskopisch nicht auflösbar. Die auf den Aufnahmen abgebildete Uhr zeigt das Alter der Kultur an. Sie verzeichnet auf dem inneren Kreis die Zahl der Tage und auf dem äußeren die Stunden. Der hier gezeigte Vorgang umfaßt einen Zeitraum von 5 Tagen und 20 Stunden. Die phasenkontrast-mikroskopischen Abb. 7-9 unterstreichen in eindrucksvoller Form, in welchem Ausmaße diese Körperchen produziert werden können.

Auf Abb. 10 ist ein schatten-gleiches Bakterium mit üblichen Abmessungen zu erkennen, welches einem kleinen Aggregat von drei parallel liegenden, normalen Stäbchen aufsitzt. Im zentralen Bereich dieses Bakteriums finden sich Körnchen aller Größen, die z. T. in etwas dichterem Plasma lagern. Abb. 11 zeigt zwei Bakterien, die durch Teilung aus einer Mutterzelle hervorgegangen sind. Die in ihrer ganzen

2 H. Ruska, G. Bringmann, I. Neckelu. G. S chu st e r, Z. wiss. Mikroskop. mikroskop. Techn. 60, 425 [1952].

3 H. K ölb e l, Z. Naturforschg. 8 b, 631 [1953].

* Abb. 1-14 s. Tafel S. 432 a-d. 
Länge dargestellte Zelle ist aufgetrieben und kontrast-arm. Das Plasma ist vakuolisiert. Diese Vakuolen sind eine Folge des Auflösungsprozesses, während die ebenfalls als Vakuolen bezeichneten rundlichen Blasen, die im Frühstadium der Vermehrung auftreten, sicher anderen Ursprungs sind. Die Blasen, welche in der nur z. T. sichtbaren Tochterzelle und vielfach auch auf Abb. 13 erscheinen, sind mit einer dünnen Membran versehene, organisierte Zellbereiche, in deren Innerem meist eine größere Anzahl kleiner Körnchen angetroffen werden. In den Vakuolen lysierender Bakterien findet man sie dagegen nicht. Ein Teil der Körnchen auf Abb. 11 liegen am Schrumpfungssaum deutlich außerhalb der Zelle. Der Austrittsort ist nicht erkenntlich. Die Trennungsstelle zwischen den beiden Bakterien ist nur durch mechanisches Reißen beim Trocknungsprozeß zu erklären. Die seitliche Ausstülpung zeigt eine durchgehende, nicht unterbrochene Kontur. Es scheint nicht ausgeschlossen, daß die Körnchen die Zellbegrenzung durchwandern können, ohne eine Läsion hervorzurufen.

Die weiteren e-optischen Abb. (12-14) zeigen intra- und extrazelluläre Körnchen in großer Zahl. Die außerhalb der Zelle liegenden finden sich nicht nur in unmittelbarer Nähe der Bakterien, sondern können allein oder zu mehreren aggregiert auch frei im Medium auftreten (Abb. 12 u. 13, Bildrand). Bei den extrazellulären handelt es sich um Körnchen mittlerer und kleinerer Größen; die größeren mit einem Durchmesser von etwa $0,5 \mu$, wie sie in geringer Zahl meist in den Bakterien zu sehen sind, haben wir dagegen nicht freiliegend angetroffen.

Wichtig scheint uns die Beobachtung, daß die auf engstem Raum zusammenlagernden Körnchen nie in „dichtester Kugelpackung“ auftreten. Soweit die Körnchen nicht übereinander lagern und durch die Projektion eine direkte Berührung vorgetäuscht wird, bleibt ein Zwischenraum frei, der für Elektronen völlig durchlässig ist (Abb. 12-14). Wir sehen hierin einen weiteren Hinweis darauf, daß das Metaphosphat-Körnchen nicht den gesamten Bereich des Granulums ausfüllt $\left(\mathrm{K} \ddot{\mathrm{O} l \mathrm{bel}}{ }^{3}\right)$.

Lichtmikroskopisch ist das Auftreten freier Granula von vielen Beobachtern gesehen und in verschiede-

4 E. W e s s e 1, Z. Tuberkulose 88, 22 [1942]. [1951].

5 G. H. Werner, Fortschr. Tbk.-Forschg. 4, 53

6 E. M. Brieger, V. E. Cosslett u. A. M. G l a u e r t, J. gen. Microbiol. 10, 294 [1954].

7 H. J. Corper u. H. C. S w e a n y, J. Bacteriol. 3, 129 [1918]. nen Richtungen gedeutet worden, wobei die unterschiedlichsten granulären Elemente unter dem Sammelbegriff „Granulum“ zusammengefaßt wurden. Neuere Arbeiten auf diesem Gebiet mit der Phasenkontrast-Methodik fehlen. Entsprechende e-optische Befunde sind spärlich. W e s s e ${ }^{4}$ spricht noch von „sporider Ausstoßung“. W e r n e r ${ }^{5}$ konnte nicht entscheiden, ob die Körnchen im Cytoplasma gelöst oder in das umgebende Medium entlassen werden. $\mathrm{R} \mathrm{u} \mathrm{s} \mathrm{k} \mathrm{a}{ }^{2}$ hat freiliegende Körnchen nicht beobachten können. B ri e g e r und Mitarbb. ${ }^{6}$ vermuten lediglich, daß diese Körper freigesetzt werden. Die meisten Autoren, welche freiliegende Granula oder Körnchen beschrieben haben, sehen in ihrem Auftreten entweder eine Phase innerhalb eines Lebenszyklus oder deuten es als Degeneration mit nachfolgender Lyse.

Eine Reihe von Untersuchern (Corper und Sweany ${ }^{7}$, Laporte ${ }^{8,9,10}$, Corper und Cohn ${ }^{11}$, $\mathrm{B}$ a isden und Yegian ${ }^{12}$ ) haben im Tuberkelbazillus ein autolytisches Agens nachgewiesen, welches nach Behandlung mit verschiedenen organischen Lösungsmitteln und unter bestimmten Milieubedingungen ( $p_{\mathrm{H}}$-Wert, Temperatur) optimal aktiviert und durch Jod oder Formaldehyd sowie Hitzebehandlung inhibiert werden kann. Die Lyse der Bakterien ist hiernach ein enzymatischer Vorgang. Für ihren Eintritt ist die Art des Nährmediums von Bedeutung. Während Corper und Cohn ${ }^{11}$ einen synthetischen, eiweiß-freien Nährboden (Wo n g-W e in z i r l) angeben, hält L a p or te $\mathrm{e}^{8}$ Glycerinkartoffel und Glycerin-Bouillon für geeignet.

Wahrscheinlich darf man die Lyse nicht als reinen Degenerationsprozeß auffassen. Ihre biologische Bedeutung geht darüber hinaus und muß in Hinblick auf mögliche Auswirkungen diskutiert werden, welche freigesetzte Mitochondrien für die Gesamtpopulation haben, die ja nicht nur eine Addition von Einzelindividuen darstellt und deren Gesamtleistung und -potenz nicht nur eine Summe der Einzelleistungen ist.

Die neuere Auffassung von den Granula als Mitochondrien hat zur Konsequenz, daß wir ihnen auf Grund ihres Gehaltes an Enzymen abbauende und

8 R. L a p or te, C. R. Soc. Biol. 136, 414 [1942].

9 R. L a p orte, C. R. [Doklady] Acad. Sci. URSS 214, 887 [1942].

10 R. L a p or te, Ann. Inst. Pasteur 69, 262 [1943].

11 H. J. Corper u. M. L. Cohn, Amer. Rev. Tubercul. 48, 443 [1943].

12 L. B a is den u. D. Yegi a n, J. Bacteriol. 45, 163 [1943]. 
synthetisierende Stoffwechselleistungen auch außerhalb der zellulären Organisation zuschreiben müssen. Schon die Erscheinung der Autolyse ist Ausdruck der Enzymtätigkeit über den Zelltod hinaus. Die Möglichkeit der Isolierung dieser Komplexe bei Erhaltung der Funktion unterstreicht ihre Autonomie. Die Fermentaktivität im umgebenden Medium im Sinne einer Milieubeeinflussung muß für das Wachstum und die Vermehrung normaler, intakter Bakterien bedeutsam sein. Wir möchten zwei Beobachtungen anführen, welche auf eine Fermentleistung extrazellulärer Mitochondrien schließen lassen:

1. Es ist eine auffällige Tatsache, daß das Angehen einer Kultur in einem synthetischen, eiweißfreien Nährmedium vom Mengenverhältnis Einsaat : Nährsubstrat abhängig ist. Beim Unterschreiten einer bestimmten Grenzkonzentration bleibt eine Vermehrung aus. Bei Einsaaten, die nur um Weniges darüber liegen, wird der Übergang in die logarithmische Phase hinausgezögert. Die Angabe eines absoluten Wertes für diese Verhältniszahlen ist nicht möglich, da die Art des Nährmediums und der physiologische Überimpfungszustand der Bakterien von maßgeblicher Bedeutung sind.

2. Beim Mycobacterium lacticola machte v. Ploth o ${ }^{13}$ folgende Beobachtung. ,Je isolierter ein Stäbchen liegt, um so länger ist die Latenzzeit bis zum Wachstumsbeginn auf dem neuen Medium. Liegen die Stäbchen relativ dicht nebeneinander, so beginnt die Teilung schneller."

Es ist hieraus $\mathrm{zu}$ ersehen, daß sich ein isolierter Keim bei seiner Vermehrung in einem frischen Nährsubstrat vor andere Aufgaben gestellt sieht, als wenn er sich mit vielen Keimen in einer Population befinden würde. Gewisse interbakterielle Stoffe oder Stoffkomplexe, die von den Bakterien selbst produziert werden, scheinen eine Rolle bei der Vermehrung zu spielen. Offenbar muß die Minimalkonzentration einer bestimmten lebensnotwendigen Substanz vorliegen oder die Möglichkeit zu ihrer Synthese gegeben sein. Die Dauer der Anpassungsphase richtet sich dann danach, ob und in welcher Zeit dieser Stoff in ausreichender Menge zur Verfügung gestellt werden kann. Bei kleineren Einsaaten diffundieren diese Stoffe frei in das umgebende Medium, ohne daß sie wegen ihrer geringen Konzentration einerseits und

13 O. v. Pl ot ho, Arch. Mikrobiol. 13, 91 [1942].

14 P. R on d o ni, Ergebn. Enzymforsch. 10, 146 [1949].

15 H. L e t tré, Naturwissenschaften 37, 335 [1950]. der geringen Keimzahl andererseits zur Wirkung gelangen könnten. Es ist unwahrscheinlich, daß es sich hierbei um relativ einfache organische Verbindungen aus dem normalen Stoffwechsel handelt. Vielmehr ist anzunehmen, daß das bessere Wachstum eines Keimes in einer Population dadurch zustande kommt, daß einzelne Keime - gemäß unserer mikroskopischen Befunde - ihre Mitochondrien entlassen, wodurch zu Gunsten anderer das für ihre Vermehrung ungünstige Nährsubstrat in geeigneter Form aufbereitet wird. In einem beimpften Nährsubstrat, in welchem sich eine kleine Anzahl mehr oder weniger isolierter Einzelkeime befinden, reicht die enzymatische Aktivität der relativ geringen Menge an freigesetzten Mitochondrien nicht aus, um die Grenzkonzentration der Substanz bereitzustellen, die für eine Vermehrung erforderlich ist. Die Zahl der Mitochondrien von in Autolyse begriffenen Keimen, die auf diese Weise „geopfert“ werden, ist ungleich größer als die der normalen. Man hat den Eindruck, als ob zahlreiche Mitochondrien auf Kosten der gesamten plasmatischen Substanz produziert werden. Die Bakterien werden hierbei völlig kontrast-arm und verblassen.

$\mathrm{Zu}$ welch weitgehenden Folgerungen über die Bedeutung strukturierter plasmatischer Elemente in der höheren Zelle man bereits früher geführt wurde, zeigt eine Bemerkung von $\mathrm{R}$ on $\mathrm{d}$ o n $\mathrm{i}^{14}$, wonach als möglich gilt, „daß kleinere, subzelluläre Einheiten oder pathologische Zellprodukte eine gewisse Autonomie erlangen, aus der Zelle austreten und in andere Zellen Eingang finden können." Diese vorsichtig formulierte Vermutung findet ihre Bestätigung durch die bedeutsamen Befunde von L e $t$ tré ${ }^{15,16,17}$ über die Aufnahme isolierter Mitochondrien aus Ascites-Tumor-Zellen in solche gleicher Art. Ein entsprechender Vorgang ist auch für die Mitochondrien der Mycobacterien in Erwägung zu ziehen.

B ö n i c k e ${ }^{18}$ fand, daß bei Zusatz von Hämin zum eiweißfreien synthetischen Nährmedium nach Lockemann auch kleinste Einsaaten zur Vermehrung kommen. Dieser Effekt ist sonst nur bei Zugabe von Serum oder Albumin erzielbar. Die im Serum vorhandenen Eiweißstoffe sind damit wichtig für die Synthese des Hämins. Die Hämine als Porphyrin-Eisenkomplexe nehmen in Gestalt der Cytochrome, Cytochrom-Oxydase, Peroxydase und Katalase eine zen-

16 H. Lettré, Naturwissenschaften 40, 25 [1953].

17 H. Le ttré, Naturwissenschaften 41, 144 [1954].

18 R. B ö n i c k e, Naturwissenschaften, 41, 378 [1954]. 
trale Stellung im Stoffwechsel ein. Es ist naheliegend, eine maßgebliche Beteiligung der Mitochondrien an ihrer Synthese anzunehmen.

Auch unter einem anderen Gesichtspunkt muß das extrazelluläre Auftreten von Granula diskutiert werden. Nach früheren Untersuchungen von $\mathrm{L}$ a porte 8,19 , Laporte und Vendrely ${ }^{20}$ und L e m b ke ${ }^{21}$ geben aus Kulturen isolierte granuläre Partikel eine Tuberkulin-Reaktion. Diese Untersuchungen gewinnen dadurch an Bedeutung, daß die Abmessungen der Granula von gleicher Größenordnung sind wie die von Corper und Cohn ${ }^{11}$ durch Filtration bestimmte Partikelgröße des bei der Autolyse freigesetzten Tuberkulins. Wenn man voraussetzt, daß eine Isolierung quantitativ möglich war, wie es den Anschein hat, dann enthalten die Mitochondrien das Tuberkulin.

In ultradünnen Schnitten von Lunge und Milz in-

19 R. L a p or te, Ann. Inst. Pasteur 71, 51 [1945].

20 R. L a p orte u. R. Ve ndrely, Bull. Soc. Chim. biol. 26, 437 [1944]. fizierter Kaninchen und Mäuse konnten B rieger und Glauert ${ }^{22}$ e-optisch eine große Anzahl von Körperchen mit einem Durchmesser von 0,2 bis $0,8 \mu$ finden, die von normalen Zelleinschlüssen unterschieden werden konnten und wahrscheinlich bakterieller Natur sind.

Diese Befunde sind für die Pathogenese der Tuberkulose von Bedeutung. Doch sind weitere Untersuchungen notwendig, um die hier genannten Stoffkomplexe den Granula zuordnen zu können.

Die Aufnahmen wurden mit der Leitz-Phasenkontrasteinrichtung und mit einem $100 \mathrm{kV}$-Siemens-Elektronenmikroskop (ÜM 100, Elmiskop I) hergestellt. Wir danken der Abt. Elektronenoptik im Wernerwerk für Meßtechnik der Siemens \& Halske AG., Berlin, insbesondere den Herren Prof. Dr. E. R u s k a und Dr. W. Th a l sowie Fräulein Dr. C. W e ichan für ihr Entgegenkommen.

21 A. L e m b k e, Zbl. Bakteriol., Parasitenkunde, Infektionskrankh. Hyg., I. Abt., Orig. 152, 239 [1947].

22 E. M. B ri eger u. A. M. Gla u e r t, Tubercle 35,80 [1954].

\title{
Zur Kinetik der Rekombinantenbildung bei T1-Bakteriophagen
}

\author{
Von Carsten Bresch und Thomas Trautner \\ Aus dem Max-Planck-Institut für physikalische Chemie, Göttingen \\ (Z. Naturforschg. 10 b, 436-440 [1955]; eingegangen am 6. April 1955)
}

\begin{abstract}
A cross is made between 2 mutants of phage T 1 with a multiplicity of infection of 4 of each type. Infected bacteria are opened during the latent period after the method described by $\mathrm{D}$ o e r m a n n, and recombinant percentages are determined. From the increase of recombinant frequency and the increase of particle number, the recombination percentage and the mating experience of the particles in the pool are calculated, making use of the equations of the Delbr ück-Visconti theory. From the linear increase of mating experience follows constant mating probability between 8 and $14 \mathrm{~min}$ after infection. It is shown in the discussion that the beginning of mating occurs very likely at $4 \mathrm{~min}$. The mating probability is constant over the entire length of the latent period, thus being independent of the pool size.
\end{abstract}

$\mathrm{D}_{\mathrm{s}}$ e genetischen Vorgänge bei der Bakteriophagen-Vermehrung wurden durch die "mating theory" von Delbrück und Visconti ${ }^{1}$ gedeutet. Den Kern dieser Theorie bildet die Annahme, daß sich das genetische Geschehen innerhalb einer Population vegetativer Partikel abspielt, wobei die eigentlichen Paarungsakte zeitbeliebig zwischen zwei beliebigen Partnern stattfinden. Diese Annahme basierte im wesentlichen auf zwei experimentellen Befunden:

1 N. Visconti u. M. Delbrück, Genetics 38 , 5 [1953].
1. Bei Infektion eines Bakteriums mit 3 genetisch verschiedenen Eltern treten in der Nachkommenschaft Rekombinanten auf, die Merkmale aller drei Eltern vereinigen. Dieses Ergebnis wurde zuerst von $\mathrm{Hershey}$ und Rotman ${ }^{2}$ an $\mathrm{T} 2$ gewonnen und später von Bresch an $\mathrm{T} 1$ bestätigt ${ }^{3}$.

2. Der Prozentsatz von Rekombinanten steigt bei T 2 im Laufe der Latenzperiode und - im

2 A. D. H e r s he y u. R. R ot m a n, Proc. nat. Acad. Sci. USA 34, 89 [1948].

3 C. B r e s c h, Ann. Inst. Pasteur 84, 157 [1953]. 